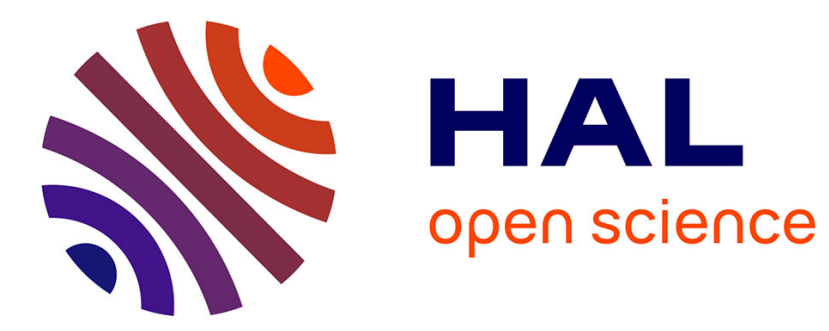

\title{
Vibration damping in low-frequency range due to structural complexity. A Model based on the theory of fuzzy structures and model parameters estimation.
}

Christian Soize

\section{- To cite this version:}

Christian Soize. Vibration damping in low-frequency range due to structural complexity. A Model based on the theory of fuzzy structures and model parameters estimation.. Computers \& Structures, 1996, 58 (5), pp.901-915. 10.1016/0045-7949(95)00207-W . hal-00770275

\section{HAL Id: hal-00770275 \\ https://hal.science/hal-00770275}

Submitted on 4 Jan 2013

HAL is a multi-disciplinary open access archive for the deposit and dissemination of scientific research documents, whether they are published or not. The documents may come from teaching and research institutions in France or abroad, or from public or private research centers.
L'archive ouverte pluridisciplinaire HAL, est destinée au dépôt et à la diffusion de documents scientifiques de niveau recherche, publiés ou non, émanant des établissements d'enseignement et de recherche français ou étrangers, des laboratoires publics ou privés. 


\title{
Vibration Damping in Low-Frequency Range Due to Structural Complexity. A Model Based on the Theory of Fuzzy Structures and Model Parameters Estimation
}

\author{
Christian Soize
}

Structures Department, ONERA, BP 72, F-92322 Chatillon Cedex, France

\section{INTRODUCTION}

The present paper concerns the low-frequency range. In order to help the understanding, we shortly recall the basic works previously developped in the area of the theory of fuzzy structures for the medium-frequency range.

In the medium-frequency range, the modeling of the "secondary" mechanical subsystems attached to the "primary" structure must generally be introduced to improve the structuralacoustics predictions of structures having an internal structural complexity. Vibration experiments yield smooth morphology for the MF frequency response functions of structures having a structural complexity. The rates of dissipation that would correspond to such smoothing are much too high to allow this phenomenon to be explained by mechanical damping alone. The apparent dissipation occuring on the primary structure is due to the energy transfered into the secondary mechanical subsystems attached to the primary structure and entering in vibration in the MF range. It is therefore the internal dynamical DOF of the secondary subsystems that are involved and a pure mass model of these susbsystems can in no case account for these phenomena. These observations mean that in order to predict the MF vibrations in the primary structure, we must model the dynamical effects that the secondary subsystems have on the primary structure.

In this way, we have developed the theory of fuzzy structures in several previous papers. We have developed this theory [1-3] with an adapted numerical analysis [4,5] which is based on the MF method [6,7] and we have presented some applications for the validation $[4,5]$. The MF method [6] that we have developed to solve forced vibration [6,7], radiation problems [7-11] and scattering problems [12,13], has been used by $R$. Vasudevan $[14,15]$ to solve radiation and scattering problems. In 1991, this theory of fuzzy structures has been revisited by V.W. Sparrow [16] who has initialized new researches in this area in the United States of America [17-24]. Recently, the author has presented [25] a survey of its theory of fuzzy structures including new results concerning probabilistic fuzzy constitutive law with spatial memory (Type II law) [26,27] and developed within the context of sound radiation and acoustic scattering problems.

In the theory of fuzzy structures, we have introduced [2] the term "master structure" to designate the "primary" structure and, in some cases, internal and/or external fluid regions, i.e. the part of the mechanical systems that is accessible to conventional modeling. The complement to the master structure with respect to the complete mechanical system is designated as the "fuzzy substructure". (In [1,2,25], we said "structural fuzzy"). It is by definition the part of the structure that is not accessible to conventional modeling because details are unknown or are imprecisely known. The fuzzy substructure consists of the secondary mechanical subsystems "attached" to the primary structure. Finally, a "fuzzy structure" is a master structure plus one or more fuzzy substructures globally called fuzzy substructure. The set of all the substructures will be called the fuzzy substructure. 
It should be noted that in the MF range, the previous works [1-5,25] yield a complete model of the dissipation due to the structural complexity and tools for solving the random equation resulting from the probabilistic model of a fuzzy structure. In this frequency range, the equations are written using all the physical DOF of the master structure and the MF method allows the mean response and the root-mean-square response to be calculated.

For some large structures with internal structural complexity, the phenomena described above, which usually appears in the MF range, can also appear in low-frequency (LF) range. This means that in the LF range in which the master structure has a modal response, the values of modal damping rates cannot be explained by mechanical damping alone, but is mainly due to the mechanical energy transfered from the master structure into the fuzzy substructure, exactly as for the MF range.

In this paper, we present a modeling of the structural dissipation due to internal structural complexity, adapted to the low frequency range for which the frequency response function of the master structure can be constructed by the modal synthesis. This vibration damping model is deduced from the theory of fuzzy structures which was previously developed by the author. Presently, this model uses only the mean part of the probabilistic fuzzy law of the fuzzy substructure [2,25]. We give an explicit model of the generalized damping matrix induced by the internal structural complexity. This generalized damping matrix depends on parameters related to the fuzzy substructure. Finally, model updating considerations are studied and an example is presented.

\section{MODELING IN THE LF RANGE FOR VIBRATION DAMPING DUE TO STRUCTURAL COMPLEXITY}

This section deals with the modeling and the mathematical formulation in the low frequency range for vibration predictions using the theory of fuzzy structures. The formulation developed is especially adapted to complex geometry. In order to simplify the formulation, we assume that there is no fluid coupled with the primary structure and consequently there is an identity between the master structure and the primary structure. The physical space $\mathbb{R}^{3}$ is referred to a cartesian reference system $O x_{1} x_{2} x_{3}$ and we will denote by $\mathbf{x}=\left(x_{1}, x_{2}, x_{3}\right)$ the generic point of $\mathbb{R}^{3}$. The geometry of the fuzzy structure and the notations are defined in Fig. 1 . The master structure is an elastic continuum which occupies the open bounded domain $D_{\text {mast }}$ of $\mathbb{R}^{3}$ with boundary $\partial D_{\text {mast }}=\Sigma_{\text {mast }} \cup \Sigma_{\text {fuz }}$. The part $\Sigma_{\text {fuz }}=\cup_{\ell=1}^{L} \Gamma_{\ell}$ is the common boundary between the fuzzy substructure and the master structure.

\subsection{Modeling of the master structure}

In the LF range, we assume that the master structure can be modeled by an inhomogeneous, anisotropic, linear viscoelastic solid continuum. Let $\mathbf{x} \mapsto \mathbf{U}(\mathbf{x}, \omega)$ be the $\mathbb{C}^{3}$-valued displacement field of the master structure defined on $D_{\text {mast }}$. Let $\mathbf{M}_{\text {mast }}, \mathbf{C}_{\text {mast }}(\omega)$ and $\mathbf{K}_{\text {mast }}$ be the mass, damping and stiffness symmetric real linear operators constructed by the variational formulation. Symmetric operator $\mathbf{M}_{\text {mast }}$ is positive definite and symmetric operators $\mathbf{C}_{\text {mast }}(\omega)$ and $\mathbf{K}_{\text {mast }}$ are positive. The non dependency on $\omega$ of $\mathbf{K}_{\text {mast }}$ is due to the fact that, in the LF range, this operator can be approximated by an operator independent of $\omega$. If it was not the case, the modal representation of the response would have no meaning and in that case an MF model should be used even if the frequency band is located in the LF range. Then, the impedance operator $\mathbf{Z}_{\text {mast }}(\omega)$ of the master structure can be written as

$$
i \omega \mathbf{Z}_{\text {mast }}(\omega)=-\omega^{2} \mathbf{M}_{\text {mast }}+i \omega \mathbf{C}_{\text {mast }}(\omega)+\mathbf{K}_{\text {mast }} .
$$


We denote the element representing the mechanical forces applied on the master structure as $\mathbf{f}_{\text {mast }}(\omega)$. Finite approximation of operators in eqn. (1) are usualy constructed using the finite element method.

\subsection{Modeling of the fuzzy substructure}

The fuzzy substructure is attached to the master structure on the part $\Sigma_{\text {fuz }}$ which can be written as

$$
\Sigma_{\mathrm{fuz}}=\cup_{\ell=1}^{L} \Gamma_{\ell} \quad, \quad \cap_{\ell=1}^{L} \Gamma_{\ell}=\emptyset,
$$

such that (1)- Boundary $\Gamma_{\ell}$ is smooth. (2)- A fuzzy substructure having a continuous boundary $[2,25]$ is attached to each part $\Gamma_{\ell}$ of $\Sigma_{\mathrm{fuz}}$. (There is no problem to extend the developments below to a fuzzy substructure having a locally discrete boundary [2,25]). (3)- There is no mechanical connection between two fuzzy substructures attached to different parts $\Gamma_{\ell}$ and $\Gamma_{\ell^{\prime}}$ with $\ell \neq \ell^{\prime}$, except the common boundary with the master structure. Consequently, there is no spatial memory effect [25] between such fuzzy substructures. A contrario, a spatial memory effect can exist inside any fuzzy substructure attached to $\Gamma_{\ell}$. (4)- A homogeneous orthotropic fuzzy substructure having possibly a spatial memory effect inside it [2,25] is attached to each part $\Gamma_{\ell}$. It should be noted that an isotropic fuzzy substructure can be modeled with the model considered above. Therefore, we use the type II fuzzy constitutive law [25] which models a fuzzy substructure with spatial memory effect. If there is no spatial memory effect, then the type II law degenerates in the type I fuzzy constitutive law [2].

Let $d s(\mathbf{x})$ be the two-dimensional area measure on $\Sigma_{\text {fuz }}$. We then have $\int_{\Gamma_{\ell}} d s(\mathbf{x})=\left|\Gamma_{\ell}\right|$, where $\left|\Gamma_{\ell}\right|$ is the area of surface $\Gamma_{\ell}$. Let $\mathbb{W}$ be the space of functions $\mathbf{x} \mapsto \mathbf{W}(\mathbf{x})=\left(W_{1}, W_{2}, W_{3}\right)$ defined on $D_{\text {mast }}$ with values in $\mathbb{C}^{3}$, having a trace $\mathbf{x} \mapsto \mathbf{w}(\mathbf{x})=\left(w_{1}, w_{2}, w_{3}\right)$ on $\Sigma_{\text {fuz }}$ which is an integrable square function for $d s$. One introduces the notation

$$
\ll \mathbf{W}, \mathbf{W}^{\prime} \gg=\int_{D_{\text {mast }}}<\mathbf{W}(\mathbf{x}), \mathbf{W}^{\prime}(\mathbf{x})>d \mathbf{x}
$$

in which $<\mathbf{W}(\mathbf{x}), \overline{\mathbf{W}^{\prime}(\mathbf{x})}>=\sum_{j=1}^{3} W_{j}(\mathbf{x}) \overline{W_{j}^{\prime}(\mathbf{x})}$ and an overline means the complex conjugate.

Let $\left[\underline{Z}^{\ell}\left(\mathbf{x}, \mathbf{x}^{\prime}, \omega\right)\right]$ be the $(3 \times 3)$ complex matrices relative to the canonical basis and modeling the mean boundary impedance of the fuzzy substructure attached to $\Gamma_{\ell}$. Recall that we only consider the mean part of the probabilistic model developped in ref. [2,25], the random fluctuation being cancelled. We have $\left[\underline{Z}^{\ell}\left(\mathbf{x}, \mathbf{x}^{\prime}, \omega\right)\right]=\left[\underline{Z}^{\ell}\left(\mathbf{x}^{\prime}, \mathbf{x}, \omega\right)\right]^{T}$ where right exponent $T$ denotes the transposition of matrix. With these notations, the mean boundary impedance operator $\underline{\mathbf{Z}}_{\text {fuz }}(\omega)$ of the fuzzy substructure is defined for any $\mathbf{W}(\omega)$ and $\mathbf{W}^{\prime}(\omega)$ in $\mathbb{W}$ by

$$
\ll \underline{\mathbf{Z}}_{\text {fuz }}(\omega) \mathbf{W}(\omega), \mathbf{W}^{\prime}(\omega) \gg=\sum_{\ell=1}^{L} \int_{\Gamma_{\ell}} \int_{\Gamma_{\ell}}<\left[\underline{Z}^{\ell}\left(\mathbf{x}, \mathbf{x}^{\prime}, \omega\right)\right] \mathbf{w}\left(\mathbf{x}^{\prime}, \omega\right), \overline{\mathbf{w}^{\prime}(\mathbf{x}, \omega)}>d s(\mathbf{x}) d s\left(\mathbf{x}^{\prime}\right)
$$

For any fixed $\omega$ in $\mathbb{R}$, complex linear operator $\underline{\mathbf{Z}}_{\text {fuz }}(\omega)$ is algebraically symmetric and can be written as

$$
i \omega \underline{\mathbf{Z}}_{\mathrm{fuz}}(\omega)=-\omega^{2} \underline{\mathbf{R}}_{\mathrm{fuz}}(\omega)+i \omega \underline{\mathbf{I}}_{\mathrm{fuz}}(\omega),
$$

in which $\underline{\mathbf{R}}_{\text {fuz }}(\omega)$ and $\underline{\mathbf{I}}_{\text {fuz }}(\omega)$ are real linear operators, algebraically symmetric, such that $\omega \underline{\mathbf{R}}_{\mathrm{fuz}}(\omega)=-\Re e\left\{i \underline{\mathbf{Z}}_{\mathrm{fuz}}(\omega)\right\}$ and $\underline{\mathbf{I}}_{\mathrm{fuz}}(\omega)=\Im m\left\{i \underline{\mathbf{Z}}_{\mathrm{fuz}}(\omega)\right\}$, operator $\underline{\mathbf{I}}_{\mathrm{fuz}}(\omega)$ being a positive operator $[2,25]$. 


\subsection{Mean operatorial equation of the master structure coupled with its fuzzy substructure}

The mean operatorial equation of the master structure coupled with its fuzzy substructure is deduced from $[2,25]$ :

$$
i \omega\left[\mathbf{Z}_{\text {mast }}(\omega)+\underline{\mathbf{Z}}_{\text {fuz }}(\omega)\right] \mathbf{U}(\omega)=\mathbf{f}_{\text {mast }}(\omega)
$$

From equations (1) and (4), we deduce that eqn. (5) can be written as

$$
\left[-\omega^{2} \mathbf{M}_{\text {mast }}+i \omega\left(\mathbf{C}_{\text {mast }}(\omega)+\underline{\mathbf{I}}_{\text {fuz }}(\omega)\right)+\mathbf{K}_{\text {mast }}-\omega^{2} \underline{\mathbf{R}}_{\text {fuz }}(\omega)\right] \mathbf{U}(\omega)=\mathbf{f}_{\text {mast }}(\omega) \quad .
$$

We will assume that the conservative part $\underline{\mathbf{R}}_{\text {fuz }}$ of the fuzzy substructure depends weakly on $\omega$ in the LF range and can directly be taken into account in the modeling of the mass and stiffness operators of the fuzzy structure. It should be noted that if this kind of modeling is usual for the LF range, it cannot be used in the MF domain for which operator $\underline{\mathbf{R}}_{\text {fuz }}(\omega)$ can be kept and generated by using the type II fuzzy law [25]). Consequently, if $\mathbf{M}_{S}$ and $\mathbf{K}_{S}$ denote the mass and stiffness linear operators of the fuzzy structure respectively (conservative part of the master structure with its fuzzy substructure), then we have

$$
-\omega^{2} \mathbf{M}_{S}+\mathbf{K}_{S}=-\omega^{2} \mathbf{M}_{\text {mast }}+\mathbf{K}_{\text {mast }}-\omega^{2} \underline{\mathbf{R}}_{\mathrm{fuz}},
$$

in which $\mathbf{M}_{S}$ is a positive-definite operator and $\mathbf{K}_{S}$ is a positive linear operator. Equations (6) and (7) yield

$$
\left[-\omega^{2} \mathbf{M}_{S}+i \omega\left(\mathbf{C}_{\text {mast }}(\omega)+\underline{\mathbf{I}}_{\mathrm{fuz}}(\omega)\right)+\mathbf{K}_{S}\right] \mathbf{U}(\omega)=\mathbf{f}_{\text {mast }}(\omega)
$$

\subsection{Model of vibration damping using type II fuzzy law}

The vibration damping generated by the internal structural complexity is due to positive operator $\underline{\mathbf{I}}_{\text {fuz }}(\omega)$. Considering equations (3) and (4), we deduce that $\underline{\mathbf{I}}_{\text {fuz }}(\omega)$ can be written as

$$
\ll \underline{\mathbf{I}}_{\text {fuz }}(\omega) \mathbf{W}(\omega), \mathbf{W}^{\prime}(\omega) \gg=\sum_{\ell=1}^{L} \int_{\Gamma_{\ell}} \int_{\Gamma_{\ell}}<\left[\underline{I}^{\ell}\left(\mathbf{x}, \mathbf{x}^{\prime}, \omega\right)\right] \mathbf{w}\left(\mathbf{x}^{\prime}, \omega\right), \overline{\mathbf{w}^{\prime}(\mathbf{x}, \omega)}>d s(\mathbf{x}) d s\left(\mathbf{x}^{\prime}\right) .
$$

In this subsection, we construct the model of real matrix $\left[\underline{I}^{\ell}\left(\mathbf{x}, \mathbf{x}^{\prime}, \omega\right)\right]$ which is deduced from the theory of fuzzy structures $[2,25]$. Since the fuzzy substructure is orthotropic, by definition [2] there exists an orthogonal $(3 \times 3)$ real matrix $[\Phi(\mathbf{x})]^{T}$ for transition from canonical basis to a local orthonormal basis $\left\{\mathbf{e}^{1}(\mathbf{x}), \mathbf{e}^{2}(\mathbf{x}), \mathbf{e}^{3}(\mathbf{x})\right\}$ related to the boundary $\Gamma_{\ell}$ at point $\mathbf{x} \in \Gamma_{\ell}$ such that, for all $\omega$ in $\mathbb{R}, \mathbf{x}$ and $\mathbf{x}^{\prime}$ in $\Gamma_{\ell}$, we have

$$
\left[\underline{I}^{\ell}\left(\mathbf{x}, \mathbf{x}^{\prime}, \omega\right)\right]=[\Phi(\mathbf{x})]\left[\underline{I}_{0}^{\ell}\left(\mathbf{x}, \mathbf{x}^{\prime}, \omega\right)\right]\left[\Phi\left(\mathbf{x}^{\prime}\right)\right]^{T},
$$

in which $\left[\underline{I}_{0}^{\ell}\left(\mathbf{x}, \mathbf{x}^{\prime}, \omega\right)\right]$ is a $(3 \times 3)$ real diagonal matrix. For all $\alpha$ and $\beta$ in $\{1,2,3\}$, we can write

$$
\left[\underline{I}_{0}^{\ell}\left(\mathbf{x}, \mathbf{x}^{\prime}, \omega\right)\right]_{\alpha \beta}=\delta_{\alpha \beta} c_{\beta}^{\ell}\left(\mathbf{x}, \mathbf{x}^{\prime}, \omega\right)
$$

in which $\delta_{\alpha \beta}=0$ if $\alpha \neq \beta$ and $\delta_{\alpha \alpha}=1$ and where $c_{\beta}^{\ell}\left(\mathbf{x}, \mathbf{x}^{\prime}, \omega\right)$ are real valued functions. We have $c_{1}^{\ell}\left(\mathbf{x}, \mathbf{x}^{\prime}, \omega\right)=c_{2}^{\ell}\left(\mathbf{x}, \mathbf{x}^{\prime}, \omega\right)=c_{3}^{\ell}\left(\mathbf{x}, \mathbf{x}^{\prime}, \omega\right)=c^{\ell}\left(\mathbf{x}, \mathbf{x}^{\prime}, \omega\right)$ for an isotropic fuzzy 
substructure. We directly deduce from the fuzzy structure theory $[2,25]$ that, for all $\beta$ in $\{1,2,3\}$, one has

$$
c_{\beta}^{\ell}\left(\mathbf{x}, \mathbf{x}^{\prime}, \omega\right)=\delta_{\Sigma_{\mathrm{fuz}}}\left(\mathbf{x}-\mathbf{x}^{\prime}\right) \underline{\nu}_{\beta}^{\ell} h_{\beta}^{\ell}(\omega),
$$

in which $\delta_{\Sigma_{\text {fuz }}}$ is such that, if $\mathbf{x}^{\prime} \in \Gamma_{\ell}$, then $\int_{\Gamma_{\ell}} \varphi(\mathbf{x}) \delta_{\Sigma_{\text {fuz }}}\left(\mathbf{x}-\mathbf{x}^{\prime}\right) d s(\mathbf{x})=\varphi\left(\mathbf{x}^{\prime}\right)$, for any complex-valued continuous function $\varphi$ defined on $\Gamma_{\ell}$ and where, for $\omega \underline{n}_{\beta}^{\ell}>1 / 4$, we have (type II fuzzy law):

$$
h_{\beta}^{\ell}(\omega)=\frac{m_{\beta}^{\ell}}{\left|\Gamma_{\ell}\right|} \frac{1}{\underline{n}_{\beta}^{\ell}} \mathcal{J}\left(\underline{\xi}_{\beta}^{\ell}, 2 \omega \underline{n}_{\beta}^{\ell}, \underline{\alpha}_{\beta}^{\ell}\right)
$$

in which, for $\tau \in[0.5,+\infty[$, positive-valued function $\mathcal{J}$ is such that

$$
\begin{gathered}
\mathcal{J}(\xi, \tau, \alpha)=\xi \tau(1-\alpha)+\alpha \mathcal{J}_{0}(\xi, \tau) \\
\mathcal{J}_{0}(\xi, \tau)=\frac{\tau^{2}}{8 \sqrt{1-\xi^{2}}}\left\{\arctan Y_{+}(\xi, \tau)-\arctan Y_{-}(\xi, \tau)\right\} \\
Y_{ \pm}(\xi, \tau)=\frac{1}{\xi \sqrt{1-\xi^{2}}}\left\{\xi^{2}+\frac{1}{2 \tau}\left(\frac{1}{\tau} \pm 2\right)\right\}
\end{gathered}
$$

For a fuzzy substructure attached to $\Gamma_{\ell}$ and for each direction $\beta$, the input mean fuzzy parameters $\underline{\nu}_{\beta}^{\ell}, \underline{n}_{\beta}^{\ell}, \underline{\xi}_{\beta}^{\ell}, \underline{\alpha}_{\beta}^{\ell}$ which appear in eqn. (12) et (13) are defined below. These parameters depend on the frequency. We have suppressed this dependency to simplify the writing. It should be noted that this frequency dependency is significant on a broad MF frequency band for which the theory of fuzzy structures has been developped (see [2,25]). Within the present context of the use of this theory to the LF range, one can be led to neglect this frequency dependency for some input parameters, for instance for $\underline{\xi}_{\beta}^{\ell}$ and $\underline{\nu}_{\beta}^{\ell}$.

(1)- Parameter $\underline{\mu}_{\beta}^{\ell} \geq 0$ is the mean equivalent mass per unit measure of the surface $\Gamma_{\ell}$ of the fuzzy substructure for direction $\beta$ introduced in the type I or II fuzzy law (see $[2,25]$ ). We can always rewritten it as

$$
\underline{\mu}_{\beta}^{\ell}=\underline{\nu}_{\beta}^{\ell} \frac{m_{\beta}^{\ell}}{\left|\Gamma_{\ell}\right|},
$$

where $m_{\beta}^{\ell} \geq 0$ is the total mass of the fuzzy substructure attached to $\Gamma_{\ell}$ and acting in direction $\beta$. Consequently, $\underline{\nu}_{\beta}^{\ell} \geq 0$ appears as the mean coefficien of participating fuzzy mass which is a dimensionless parameter.

(2)- Parameter $\underline{n}_{\beta}^{\ell}>0$ is the mean modal density of the fuzzy substructure attached to $\Gamma_{\ell}$ for direction $\beta$. A great attention must be addressed for calculating this modal density as it is explained in Subsection 2.6.

(3)- Parameter $\underline{\xi}_{\beta}^{\ell}>0$ is the mean rate of internal dissipation of the fuzzy substructure attached to $\Gamma_{\ell}$ for direction $\beta$. Generally, we have $\underline{\xi}_{\beta}^{\ell} \ll 1$ and typical values are of the order of 0.001 to 0.01 .

(4)- Finally, $\underline{\alpha}_{\beta}^{\ell}$ is the mean equivalent coupling factor (related to the spatial memory effect) of the fuzzy substructure attached to $\Gamma_{\ell}$ for direction $\beta$. This parameter is such that $0 \leq \underline{\alpha}_{\beta}^{\ell} \leq 1$. If $\underline{\alpha}_{\beta}^{\ell}=1$ the model corresponds to the type I fuzzy law. In the other cases, it is the type II fuzzy law [25]. 
Figures 2. (a) and (b) show the graph of function $\mathcal{J}(\xi, \tau, \alpha)$ defined by eqn. (14) (in $\log _{10}$ scale) in versus of parameter $\tau$ (in $\log _{10}$ scale) for different values of $\xi$ and for $\alpha$ equal to 1 (type I law) (Figure 2. (a)) and 0.1 (type II law), (Figure 2. (b)).

\subsection{Asymptotic behavior of the vibration-damping model}

It can easily be verified that for fixed $\xi$, if $\tau \rightarrow+\infty$ then

$$
\mathcal{J}(\xi, \tau, \alpha) \sim \xi \tau(1-\alpha)+\frac{\alpha \tau}{4 \xi}
$$

Practically, equation (18) can be used if $\tau^{-1 / 2} \ll \xi \leq 0.1$. Similarly, for fixed $\tau$, if $\xi \rightarrow 0$, then

$$
\mathcal{J}(\xi, \tau, \alpha) \sim \xi \tau(1-\alpha)+\frac{\pi}{8} \tau^{2} \alpha
$$

Equation (19) can be used if $2 \tau \xi^{2} \ll\left|\tau^{-1} \pm 2\right|$.

\subsection{Example: Case of a fuzzy substructure simulated with simple linear oscillators}

A fuzzy substructure can be made with any elastic continuum, but it is interesting to study the case of a fuzzy substructure simulated with a great number of simple linear oscillators because an explicit expression of fuzzy parameters can be obtained in that case [4]. Let $\Gamma_{\ell}$ be a bounded smooth surface in physical space $\mathbb{R}^{3}$. A local orthonormal basis $\left\{\mathbf{e}^{1}(\mathbf{x}), \mathbf{e}^{2}(\mathbf{x}), \mathbf{e}^{3}(\mathbf{x})\right\}$ can be attached at each point $\mathbf{x} \in \Gamma_{\ell}$ such that $\mathbf{e}^{3}(\mathbf{x})$ is the unit normal to $\Gamma_{\ell}$ at point $\mathbf{x}$. One assumes that the fuzzy substructure is homogeneous and orthotropic acting only in direction defined by $\mathbf{e}^{3}$, with no spatial memory effect $\left(\underline{\alpha}_{3}^{\ell}=1\right)$. One has proved [4] that, in a frequency band of width $\Delta \omega$, such a fuzzy substructure can be simulated with a discrete system having a great number $N_{\ell}$ of simple linear oscillators. These oscillators act in direction defined by $\mathbf{e}^{3}$ and are randomly distributed over $\Delta \omega \times \Gamma_{\ell}$ with an uniform probability distribution. Let $M_{j}^{\ell}$ and $\xi_{j}^{\ell}$ be the mass and damping rate of the oscillator number $j$ with $j \in\left\{1, \ldots, N_{\ell}\right\}$. We then have $c_{1}^{\ell}=c_{2}^{\ell}=0$ and $c_{3}^{\ell}$ is given by equations (12)-(17) in which mean equivalent coupling factor $\underline{\alpha}_{3}^{\ell}$ is equal to 1 , mean modal density $\underline{n}_{3}^{\ell}$ is given by

$$
\underline{n}_{3}^{\ell}=\sqrt{N_{\ell}} / \Delta \omega,
$$

and the mean rate of internal dissipation is such that

$$
\underline{\xi}_{3}^{\ell}=\frac{1}{N_{\ell}} \sum_{j=1}^{N_{\ell}} \xi_{j}^{\ell}
$$

If we define the mean mass of one oscillator by the formula

$$
m_{0}^{\ell}=m_{3}^{\ell} / N_{\ell} \quad, \quad m_{3}^{\ell}=\sum_{j=1}^{N_{\ell}} M_{j}^{\ell}
$$

in which $m_{3}^{\ell}$ is the total mass of the fuzzy substructure attached to $\Gamma_{\ell}$ and acting in direction defined by $\mathbf{e}^{3}$, then the mean equivalent mass per unit measure of the surface $\Gamma_{\ell}$ is given by

$$
\underline{\mu}_{3}^{\ell}=\frac{m_{0}^{\ell} \sqrt{N_{\ell}}}{\left|\Gamma_{\ell}\right|}=\frac{1}{\sqrt{N_{\ell}}} \frac{m_{3}^{\ell}}{\left|\Gamma_{\ell}\right|} .
$$


From eqn. (17), we deduce that the dimensionless mean coefficient $\underline{\nu}_{3}^{\ell}$ of participating fuzzy mass can be written as

$$
\underline{\nu}_{3}^{\ell}=\frac{1}{\sqrt{N_{\ell}}}
$$

The calculation of these parameters is illustrated in Figure 3. It should be noted that $\underline{n}_{3}^{\ell}$ is not equal to $N_{\ell} / \Delta \omega$ but is given by eqn. (20). The reason can easily be understood looking Figure 3 and reading its legend.

\section{EIGENMODES AND GENERALIZED EQUATION OF VIBRATIONS}

The eigenfrequencies $\Omega$ and the eigenmodes $\Psi$ belonging to the set $\mho_{S}$ of admissible displacement fields of the fuzzy structure are solutions of the spectral problem

$$
\mathbf{K}_{S} \Psi=\Omega^{2} \mathbf{M}_{S} \Psi
$$

where $\mathbf{M}_{S}$ et $\mathbf{K}_{S}$ are the linear operators defined by eqn. (7). The spectrum is countable and one has $0 \leq \Omega_{1} \leq \Omega_{2} \leq \ldots$. It should be noted that for the three-dimensional elasticity case, the first eigenvalue can be equal to zero with a multiplicity between 1 and 6 . The eigenmodes $\left\{\Psi^{j}\right\}_{j \geq 1}$ form a complete set in $\mathbb{Y}_{S}$ and one has

$$
\begin{aligned}
& \ll \mathbf{M}_{S} \Psi^{j^{\prime}}, \Psi^{j} \gg=\mathcal{M}_{j} \delta_{j j^{\prime}}, \\
& \ll \mathbf{K}_{S} \boldsymbol{\Psi}^{j^{\prime}}, \boldsymbol{\Psi}^{j} \gg=\mathcal{M}_{j} \Omega_{j}^{2} \delta_{j j^{\prime}},
\end{aligned}
$$

where $\mathcal{M}_{j}$ are the generalized masses which depend on the normalization of eigenmodes. In LF range, we are interested in the approximation of $\mathbf{U}(\omega)$ in a subspace $\mathbb{V}_{N_{S}}$ of $\mathbb{V}_{S}$ spanned by $N_{S}$ eigenmodes. In order to simplify the notations, we renumber from 1 to $N_{S}$ the $N_{S}$ eigenmodes considered. Consequently, $\mathbb{Y}_{N_{S}}$ is spanned by $\left\{\boldsymbol{\Psi}^{1}, \ldots, \boldsymbol{\Psi}^{N_{S}}\right\}$ and the approximation $\mathbf{U}_{N_{S}}(\omega)$ of $\mathbf{U}(\omega)$ relatively to $\mathbb{Y}_{N_{S}}$ can be written as

$$
\mathbf{U}_{N_{S}}(\omega)=\sum_{j=1}^{N_{S}} Q_{j}(\omega) \Psi^{j}
$$

where $\mathbf{Q}(\omega)=\left(Q_{1}(\omega), \ldots, Q_{N_{S}}(\omega)\right) \in \mathbb{C}^{N_{S}}$ is the vector of the generalized coordinates. Let $\left[\mathcal{M}_{S}\right]$ and $\left[\Omega_{S}\right]$ be the $\left(N_{S} \times N_{S}\right)$ diagonal real matrices such that

$$
\left[\mathcal{M}_{S}\right]_{j j^{\prime}}=\mathcal{M}_{j} \delta_{j j^{\prime}} \quad, \quad\left[\Omega_{S}\right]_{j j^{\prime}}=\Omega_{j} \delta_{j j^{\prime}} .
$$

Let $\left[\mathcal{C}_{\text {mast }}(\omega)\right]$ and $\left[\underline{\mathcal{C}}_{\text {fuz }}(\omega)\right]$ be the $\left(N_{S} \times N_{S}\right)$ real matrices such that

$$
\begin{aligned}
{\left[\mathcal{C}_{\text {mast }}(\omega)\right]_{j j^{\prime}} } & =\ll \mathbf{C}_{\text {mast }}(\omega) \Psi^{j^{\prime}}, \Psi^{j} \gg \\
{\left[\underline{\mathcal{C}}_{\text {fuz }}(\omega)\right]_{j j^{\prime}} } & =\ll \underline{\mathbf{I}}_{\text {fuz }}(\omega) \Psi^{j^{\prime}}, \Psi^{j} \gg
\end{aligned}
$$

Symmetric matrices $\left[\mathcal{C}_{\text {mast }}(\omega)\right]$ and $\left[\underline{\mathcal{C}}_{\text {fuz }}(\omega)\right]$ are positive and called the generalized damping matrices of the master structure and due to the structural complexity respectively. From eqns. (8), (27)-(32), we deduce that $\mathbf{Q}(\omega)$ verifies the following linear system of equations on $\mathbb{C}^{N_{S}}$

$$
\left[-\omega^{2}\left[\mathcal{M}_{S}\right]+i \omega\left(\left[\mathcal{C}_{\text {mast }}(\omega)\right]+\left[\underline{\mathcal{C}}_{\text {fuz }}(\omega)\right]\right)+\left[\mathcal{M}_{S}\right]\left[\Omega_{S}\right]^{2}\right] \mathbf{Q}(\omega)=\mathbf{F}(\omega)
$$


in which $\mathbf{F}(\omega)=\left(F_{1}(\omega), \ldots, F_{N_{S}}(\omega)\right) \in \mathbb{C}^{N_{S}}$ is the vector of external generalized forces induced by $\mathbf{f}_{\text {mast }}(\omega)$. Herein, we assume that $\mho_{N_{S}}$ is spanned only by elastic eigenmodes (the eigenmodes associated with null eigenfrequencies do not belong to $\mathbb{Y}_{N_{S}}$ ). Consequently, the $\left(N_{S} \times N_{S}\right)$ complex matrix of the left-hand side member of eqn. (33) is invertible for all $\omega$ in $\mathbb{R}$ and the generalized frequency response function (GFRF) $\omega \mapsto[\mathcal{H}(\omega)]$ from $\mathbb{R}$ into the $\left(N_{S} \times N_{S}\right)$ complex symmetric matrices can be written as

$$
[\mathcal{H}(\omega)]=\left[-\omega^{2}\left[\mathcal{M}_{S}\right]+i \omega\left(\left[\mathcal{C}_{\text {mast }}(\omega)\right]+\left[\underline{\mathcal{C}}_{\text {fuz }}(\omega)\right]\right)+\left[\mathcal{M}_{S}\right]\left[\Omega_{S}\right]^{2}\right]^{-1}
$$

\section{MODEL OF THE GENERALIZED DAMPING MATRIX DUE TO STRUCTURAL COMPLEXITY}

From eqns. (9)-(12), we deduce that $\left[\underline{\mathcal{C}}_{\text {fuz }}(\omega)\right]$ defined by eqn. (32) can be written as

$$
\left[\underline{\mathcal{C}}_{\mathrm{fuz}}(\omega)\right]=\sum_{\ell=1}^{L} \sum_{\beta=1}^{3} \underline{\nu}_{\beta}^{\ell}\left[\underline{\mathcal{C}}_{\mathrm{fuz}}^{\ell \beta}(\omega)\right],
$$

in which positive symmetric $\left(N_{S} \times N_{S}\right)$ real matrices $\left[\underline{\mathcal{C}}_{\text {fuz }}^{\ell \beta}(\omega)\right]$ are such that

$$
\left[\underline{\mathcal{C}}_{\text {fuz }}^{\ell \beta}(\omega)\right]_{j j^{\prime}}=h_{\beta}^{\ell}(\omega) \int_{\Gamma_{\ell}} b_{\beta}^{j}(\mathbf{x}) b_{\beta}^{j^{\prime}}(\mathbf{x}) d s(\mathbf{x})
$$

with $h_{\beta}^{\ell}(\omega)$ given by eqn. (13) and where, for $j \in\left\{1, \ldots, N_{S}\right\}$, vectors $\mathbf{b}^{j}(\mathbf{x})=\left(b_{1}^{j}(\mathbf{x})\right.$, $\left.b_{2}^{j}(\mathbf{x}), b_{3}^{j}(\mathbf{x})\right) \in \mathbb{R}^{3}$ are defined by the relation

$$
\mathbf{b}^{j}(\mathbf{x})=[\Phi(\mathbf{x})]^{T} \boldsymbol{\psi}^{j}(\mathbf{x})
$$

where $\boldsymbol{\psi}^{j}$ is the trace of $\Psi^{j}$ on the boundary $\Gamma_{\ell}$. Using the local basis $\left\{\mathbf{e}^{1}(\mathbf{x}), \mathbf{e}^{2}(\mathbf{x}), \mathbf{e}^{3}(\mathbf{x})\right\}$ at point $\mathbf{x} \in \Gamma_{\ell}$, which has been introduced in Subsection 2.4, vector $\mathbf{b}^{j}(\mathbf{x})$ defined by eqn. (37) can be written as

$$
b_{\beta}^{j}(\mathbf{x})=<\mathbf{e}^{\beta}(\mathbf{x}), \boldsymbol{\psi}^{j}(\mathbf{x})>\quad, \quad \beta \in\{1,2,3\} .
$$

\section{MODEL PARAMETERS ESTIMATION}

Equations (13)-(16) and (35)-(38) completely define a model of the generalized damping matrix due to structural complexity. The problem studied in this section concerns the updating of parameters $\underline{\nu}_{\beta}^{\ell}$. 


\subsection{Strategy used for model updating}

The objective is to update the model defined by eqns. (35)-(38) in a low-frequency band $B=\left[\omega_{\text {inf }}, \omega_{\text {sup }}\right]$ with $\omega_{\text {inf }} \geq 0$. We assume that all the parameters are known in this model, except the dimensionless mean coefficients $\underline{\nu}_{\beta}^{\ell}$ of participating fuzzy mass. Generally, parameters $\underline{\nu}_{\beta}^{\ell}$ depend on frequency $\omega$ (see $[2,4,25]$ ). In some cases, these parameters can be independent of $\omega$. In the LF range, if there is such a dependence with the frequency, band $B$ can always be written as $B=\cup_{k} B_{k}$ with $\cap_{k} B_{k}=\emptyset$ such that $\underline{\nu}_{\beta}^{\ell}(\omega)$ can be approximated by a constant $\underline{\nu}_{\beta}^{\ell k}$ over each subinterval $B_{k}$. For all $\omega \in B$, one can then write

$$
\underline{\nu}_{\beta}^{\ell}(\omega)=\sum_{k} \mathbf{1}_{B_{k}}(\omega) \underline{\nu}_{\beta}^{\ell k}
$$

in which $\underline{\nu}_{\beta}^{\ell k}$ is a positive constant. Consequenly, the basic problem is to update parameters $\underline{\nu}_{\beta}^{\ell k}$ for a fixed subinterval $B_{k}$. This is this problem studied in the present section.

Relatively to the fixed LF band $B$, we will assume that:

(1)- The number $N_{S}$ of eigenmodes is fixed.

(2)- The $N_{S}$ eigenmodes and eigenfrequencies of the conservative part of the fuzzy structure (master structure with its fuzzy substructure) are assumed to be known. This means, for instance, that a finite element model has been developed and updated using experiments. Therefore, we will denote the updated eigenmodes and eigenfrequencies as $\left\{\widehat{\boldsymbol{\Psi}}_{1}, \ldots, \widehat{\boldsymbol{\Psi}}_{N_{S}}\right\}$ and $\left\{\widehat{\Omega}_{1}, \ldots, \widehat{\Omega}_{N_{S}}\right\}$ respectively.

(3)- Denoting the total generalized damping matrix as

$$
\left[\mathcal{C}_{\text {tot }}(\omega)\right]=\left[\mathcal{C}_{\text {mast }}(\omega)\right]+\left[\underline{\mathcal{C}}_{\text {fuz }}(\omega)\right]
$$

we will assume that for all $\omega$ in $B$, an estimation $\left[\widehat{\mathcal{C}}_{\text {tot }}(\omega)\right]$ of $\left[\mathcal{C}_{\text {tot }}(\omega)\right]$ has been constructed. This kind of estimation can result from an usual identification based on the knowledge of estimated eigenmodes and additional appropriate experiments. In addition, we suppose that a mechanical model allows matrix $\left[\mathcal{C}_{\text {mast }}(\omega)\right]$ to be constructed for all $\omega$ in $B$ and updated for providing an estimation $\left[\widehat{\mathcal{C}}_{\text {mast }}(\omega)\right]$. It should be noted that if some experiments can be realized on the master structure without its fuzzy substructure, then the model of $\left[\mathcal{C}_{\text {mast }}(\omega)\right]$ can fully be updated. If not, then this model is only partially updated due to the use of the updated eigenmodes.

(4)- Finally, from the above point, we deduce an estimation $\left[\widehat{\mathcal{C}}_{\text {fuz }}(\omega)\right]$ of $\left[\underline{\mathcal{C}}_{\text {fuz }}(\omega)\right]$ for all $\omega$ in $B$, given by

$$
\left[\widehat{\mathcal{C}}_{\text {fuz }}(\omega)\right]=\left[\widehat{\mathcal{C}}_{\text {tot }}(\omega)\right]-\left[\widehat{\mathcal{C}}_{\text {mast }}(\omega)\right]
$$

Consequently, the problem related to the model parameters estimation consists in estimating constant parameters $\underline{\nu}_{\beta}^{\ell k}$ over frequency subband $B_{k}$, with the objective to minimize, over $B_{k}$, the distance between the known function $\omega \mapsto\left[\widehat{\mathcal{C}}_{\text {fuz }}(\omega)\right]$ and the model function $\omega \mapsto$ $\left[\underline{\mathcal{C}}_{\text {fuz }}(\omega)\right]=\sum_{\ell=1}^{L} \sum_{\beta=1}^{3} \underline{\nu}_{\beta}^{\ell k}\left[\underline{\mathcal{C}}_{\text {fuz }}^{\ell \beta}(\omega)\right]$ defined by eqn. (35) in which matrices $\left[\underline{\mathcal{C}}_{\text {fuz }}^{\ell \beta}(\omega)\right]$ are defined by eqn. (36). 


\subsection{Definitio of an objective function}

Let $\|A\|_{E}$ be the Hilbert-Schmidt norm of any real $(n \times m)$ matrix $[A]$ such that

$$
\|A\|_{E}=\left(\operatorname{tr}\left\{[A]^{T}[A]\right\}\right)^{1 / 2}=\left(\sum_{j=1}^{n} \sum_{k=1}^{m}[A]_{j k}^{2}\right)^{1 / 2} .
$$

Let $\boldsymbol{\nu}^{k}$ be the vector in $\mathbb{R}^{n}$ of all the non identically null parameters $\underline{\nu}_{\beta}^{\ell k}$. One has $n \leq 3 L$ and assume that $n \geq 1$. One introduces the objective function $\boldsymbol{\nu}^{k} \mapsto \mathrm{j}_{\mathrm{c}}\left(\boldsymbol{\nu}^{k}\right)$ from $\mathbb{R}^{n}$ into $\mathbb{R}^{+}$ such that

$$
\mathrm{j}_{\mathrm{c}}\left(\boldsymbol{\nu}^{k}\right)=\int_{\omega \in B_{k}}\left\|\left[\widehat{\underline{\mathcal{C}}}_{\mathrm{fuz}}(\omega)\right]-\sum_{\ell=1}^{L} \sum_{\beta=1}^{3} \underline{\nu}_{\beta}^{\ell k}\left[\underline{\mathcal{C}}_{\mathrm{fuz}}^{\ell \beta}(\omega)\right]\right\|_{E}^{2} P(d \omega)
$$

in which matrices $\left[\underline{\mathcal{C}}_{\text {fuz }}^{\ell \beta}(\omega)\right]$ are defined by eqn. (36) and where $P(d \omega)$ is a weighting bounded positive measure with support $B_{k}$. Weighting measure $P(d \omega)$ can be defined in different ways. For instance, the following representations can be used.

(1)- GFRF updating on discrete frequencies. In this case one has

$$
P(d \omega)=\sum_{j} p_{j} \delta_{0}\left(\omega-\omega_{j}\right)
$$

where $\omega_{j}$ 's are some values of frequency lying in band $B_{k}, p_{j}$ 's are positive weights and $\delta_{0}$ is the Dirac measure at origine of $\mathbb{R}$.

(2)- GFRF updating on subintervals of frequency. The weighting measure is then written as

$$
P(d \omega)=\sum_{j} \frac{p_{j}}{\left|\Delta \omega_{j}\right|} \mathbf{1}_{\Delta \omega_{j}}(\omega) d \omega
$$

in which $p_{j}$ 's are positive weights, $\Delta \omega_{j}$ are subintervals included in $B_{k}$ and $\left|\Delta \omega_{j}\right|$ is the width of $\Delta \omega_{j}$.

(3)- GFRF updating with any weighting function. It is the generalization of eqn. (45). The measure is then written as

$$
P(d \omega)=p(\omega) d \omega,
$$

where $\omega \mapsto p(\omega)$ is any real-valued function integrable over $B_{k}$ such that $p(\omega) \geq 0$ for all $\omega$ in $B_{k}$.

\subsection{Constrained optimization problem}

From Subsection 2.4, we deduce that the variables $\underline{\nu}_{\beta}^{\ell k}$ must be restricted to positive values. Therefore, we introduce the set $\mathbb{P}$ of admissible parameters such that

$$
\mathbb{P}=\left\{\boldsymbol{\nu}^{k} \in \mathbb{R}^{n} \quad ; \quad \underline{\nu}_{\beta}^{\ell k} \geq 0 \quad, \quad \forall \ell \quad, \quad \forall \beta\right\} .
$$

Estimation $\widehat{\boldsymbol{\nu}}^{k}$ of $\boldsymbol{\nu}^{k}$ is the solution of the following constrained optimization problem

$$
\min _{\boldsymbol{\nu}^{k} \in \mathbb{P}} \mathrm{j}_{\mathrm{c}}\left(\boldsymbol{\nu}^{k}\right)
$$


Since objective function $\mathrm{j}_{\mathrm{c}}$ is a differentiable convex function and $\mathbb{P}$ is a convex set, the quadratic programming problem defined by eqn. (48) has a unique solution $\widehat{\boldsymbol{\nu}}^{k}$. This solution is the local minimum which is also the global minimum [28]. To solve the problem defined by eqn. (48), the expression of the gradient of the objective function can be useful. It can easily be verified that we have

$$
\frac{\partial}{\partial \underline{\nu}_{\beta}^{\ell k}} \mathrm{j}_{\mathrm{c}}\left(\boldsymbol{\nu}^{k}\right)=-2\left\{g_{\ell \beta}^{k}-\sum_{\ell^{\prime}=1}^{L} \sum_{\beta^{\prime}=1}^{3} A_{\ell \beta, \ell^{\prime} \beta^{\prime}}^{k} \underline{\nu}_{\beta^{\prime}}^{\ell^{\prime} k}\right\}
$$

in which

$$
\begin{gathered}
g_{\ell \beta}^{k}=\int_{\omega \in B_{k}} \operatorname{tr}\left\{\left[\underline{\mathcal{C}}_{\text {fuz }}^{\ell \beta}(\omega)\right]\left[\widehat{\mathcal{C}}_{\text {fuz }}(\omega)\right]\right\} P(d \omega), \\
A_{\ell \beta, \ell^{\prime} \beta^{\prime}}^{k}=\int_{\omega \in B_{k}} \operatorname{tr}\left\{\left[\underline{\mathcal{C}}_{\text {fuz }}^{\ell \beta}(\omega)\right]\left[\underline{\mathcal{C}}_{\text {fuz }}^{\ell^{\prime} \beta^{\prime}}(\omega)\right]\right\} P(d \omega) .
\end{gathered}
$$

\section{VALIDATION WITH A SIMPLE EXAMPLE}

One considers a fuzzy structure whose the geometry is defined in Figure 4.

\subsection{Master structure}

It is a simply supported beam with a constant cross-sectional area, length $20 \mathrm{~m}$, total mass $20000 \mathrm{Kg}$, Young's modulus $2.1 \times 10^{11} \mathrm{~N} / \mathrm{m}^{2}$, bending inertia $0.001 \mathrm{~m}^{4}$ and a constant structural damping rate 0.01 . The frequency band of analysis is $B=[0,100] \mathrm{Hz}$. The beam is excited in bending mode in $\left(x_{1}, x_{3}\right)$ plane by a force applied at $x_{1}=9$, with a unit flat spectrum over all the band $B$ of bandwidth $\Delta \omega=2 \pi \times 100 \mathrm{rad} / \mathrm{s}$.

\subsection{Fuzzy substructure}

There are two fuzzy substructures attached to the beam on $\Gamma_{1}=\left\{x_{1} ; 4 \leq x_{1} \leq 8\right\}$ and $\Gamma_{2}=\left\{x_{1} ; 12 \leq x_{1} \leq 18\right\}$. Each fuzzy substructure is made with a great number of simple linear oscillators acting in direction $x_{3}$. All the oscillators have the same mass $m_{0}=1.8 \mathrm{Kg}$ and the same damping date $\underline{\xi}=0.002$. There are $N_{1}=484$ oscillators on $\Gamma_{1}$ and $N_{2}=729$ on $\Gamma_{2}$. The eigenfrequency and the location of the attachment point on the beam of each oscillator are uniformly distributed and generated using the method explained in Figure 3. Figure 5 shows the result of this generation for the present example. Each point represents an oscillator inside the fuzzy substructures.

\subsection{Equations for the master structure with no fuzzy substructure}

The transversal displacement of the beam is denoted as $x_{1} \mapsto U_{3}\left(\omega, x_{1}\right)$. The equations allowing field $U_{3}$ of the master structure (with no fuzzy substructure) to be calculated are usually written in the frequency domain using the eigenmodes $\left\{x_{1} \mapsto \varphi^{j}\left(x_{1}\right)\right\}$ and the eigenfrequencies $\Omega_{\text {mast }, j}$ of the beam, which are explicitly known in this case. The first values of $\Omega_{\text {mast }, j}$ are given in Table 1 . We have $\Omega_{\text {mast }, 1}=1.8 \mathrm{~Hz}$ and $\Omega_{\text {mast }, 10}=180 \mathrm{~Hz}$. 


\subsection{Equations for the fuzzy structure}

The equations of movements of the fuzzy structure (beam with linear oscillators described in Subsections 6.1 and 6.2) are written in the frequency domain using the projection of $x_{1} \mapsto U_{3}\left(\omega, x_{1}\right)$ on the independent functions $\left\{x_{1} \mapsto \varphi^{j}\left(x_{1}\right), j=1, \ldots, 10\right\}$. In the present case, all the degrees of freedom ( $\left.N_{1}+N_{2}=1213 \mathrm{DOF}\right)$ associated with the linear oscillators can explicitly be eliminated from the equations of the fuzzy structure.

\subsection{Updated eigenfrequencies and eigenmodes of the fuzzy structure}

The eigenfrequencies $\Omega_{j}$ and the eigenmodes $\Psi_{3}^{j}$ of the fuzzy structure, introduced in Section 3 , are numerically calculated using the equations evoked in Subsection 6.4. In this case, we have $\widehat{\Omega}_{j}=\Omega_{j}$ and $\widehat{\Psi}_{3}^{j}=\Psi_{3}^{j}$ where $\widehat{\Omega}_{j}$ and $\widehat{\Psi}_{3}^{j}$ are the updated values introduced in Subsection 5.2. Table 1 yields the values of eigenfrequencies $\Omega_{\text {mast }, j}$ of the master structure (with no fuzzy substructure) and eigenfrequencies $\Omega_{j}$ of the fuzzy structure (master structure with its two substructures).

\begin{tabular}{|c|c|}
\hline $\begin{array}{c}\text { Master structure } \\
\Omega_{\text {mast }, j}(\mathrm{~Hz})\end{array}$ & $\begin{array}{c}\text { Fuzzy structure } \\
\Omega_{j}(\mathrm{~Hz})\end{array}$ \\
\hline 1.8 & 1.7 \\
7.2 & 6.6 \\
16.2 & 15.5 \\
28.8 & 27.3 \\
45.0 & 43.1 \\
64.8 & 62.6 \\
88.2 & 92.7 \\
115.2 & 117.9 \\
\hline
\end{tabular}

Table 1 : Eigenfrequencies in Hertz of the master structure and the fuzzy structure

The total mass of the fuzzy substructure is $2183 \mathrm{Kg}$ which must be compared to the 20000 $\mathrm{Kg}$ of the master structure. Table 1 shows that each $\Omega_{j}$ is closed to the corresponding $\Omega_{\text {mast }, j}$.

\subsection{Calculation of the response of the fuzzy structure}

The equations of the fuzzy structure introduced in Subsection 6.4 are projected on the eigenmodes $\left\{\Psi_{3}^{j}\left(x_{1}\right), j=1, \ldots, 10\right\}$ calculated in Subsection 6.5. The response $\left(\omega, x_{1}\right) \mapsto$ $U_{3}\left(\omega, x_{1}\right)$ is then computed on the band $B$, frequency per frequency, with a frequency resolution of $0.05 \mathrm{~Hz}$. 


\subsection{Use of the fuzzy structures theory}

Each fuzzy substructure described in subsection 6.2 can be simulated with a homogeneous and orthotropic fuzzy substructure acting only in $x_{3}$ direction. There is no spatial memory effect and therefore one has $\underline{\alpha}_{3}^{1}=\underline{\alpha}_{3}^{2}=1$. From eqns. (20)-(23), we deduce that $\underline{n}_{3}^{1}=0.035$ $(\mathrm{rad} / \mathrm{s})^{-1}, \underline{n}_{3}^{2}=0.043(\mathrm{rad} / \mathrm{s})^{-1}, \underline{\xi}_{3}^{1}=\underline{\xi}_{3}^{2}=0.002, m_{0}^{1}=m_{0}^{2}=m_{0}=1.8, m_{3}^{1}=871$, $m_{3}^{2}=1312$. Fiqure 6 shows the graph of functions $\omega \mapsto h_{3}^{1}(\omega)$ and $\omega \mapsto h_{3}^{2}(\omega)$ defined by eqn. (13). Finally, we will assume that, for each fuzzy substructure, the mean coefficient of participating fuzzy mass $\underline{\nu}_{3}^{\ell}$ is a constant independent of the frequency over band $B$. From eqn. (39) and for $\ell=1,2$, we then deduce that $\underline{\nu}_{3}^{\ell}$ is a constant for all $\omega$ in $B$. Therefore, there are only two scalar parameters: $\underline{\nu}_{3}^{1}$ related to $\Gamma_{1}$ and $\underline{\nu}_{3}^{2}$ to $\Gamma_{2}$, which must be updated. For that, eqn. (46) is used with $p(\omega)=1$ for all $\omega$ in $B$. The numerical solving of the constrained optimization problem defined by eqn. (48) yields the following estimation:

$$
\widehat{\underline{\nu}}_{3}^{1}=0.0466 \quad, \quad \widehat{\underline{\nu}}_{3}^{2}=0.0386 .
$$

For this case, the theoretical values are known and given by eqn. (25), i.e. by

$$
\underline{\nu}_{3}^{1}=0.0455 \quad, \quad \underline{\nu}_{3}^{2}=0.0370 \quad .
$$

It should be noted that the values given by the constrained optimization problem (eqn. (52)) are closed to the theoretical values given by eqn. (53). This result validates the theory proposed within the context of the present example.

\subsection{Results}

The results shown on Figures 7 and 8 concern the transversal acceleration of the beam at point $x_{1}=9$ (Figures 7 (a)-(c)) and at $x_{1}=14$ (Figures 8 (a)-(c)). On these figures, (a), (b) and (c) represent the modulus (in $\mathrm{dB}$ ), the real part and the imaginary part (in linear scale) of the acceleration, respectively. On each Figure 7 or 8, three results are presented.

(1)- The first one concerns the response of the master structure with no fuzzy substructure (dashed line) calculated with the equations of subsection 6.3.

(2)- The second result represents the response of the fuzzy structure, i.e. the beam with its oscillators (irregular solid line) calculated with the method of Subsection 6.6.

(3)- The third and last result is the response of the fuzzy structure calculated using the fuzzy structures theory (smoothed solid line), i.e. with eqns. (29), (33), (35)-(38), where $\underline{\nu}_{3}^{1}$ and $\underline{\nu}_{3}^{2}$ in eqn. (35) are the updated values $\underline{\underline{\nu}}_{3}^{1}$ and $\widehat{\underline{\nu}}_{3}^{2}$ defined by eqn. (52).

Figures 7 and 8 show that:

(1)- When the frequency increases, the vibration damping due to structural complexity increases. This result is also illustrated on Figure 9 which shows seven lines. Each line is associated with the index $j$ of the eigenmode $\Psi^{j}$ of the fuzzy structure and represents the function

$$
\omega \mapsto \underline{\xi}_{\text {fuz }, j j}(\omega)=\left\{\left[\mathcal{M}_{S}\right]^{1 / 2}\left[\Omega_{S}\right]\left[\underline{\mathcal{C}}_{\text {fuz }}(\omega)\right]\left[\Omega_{S}\right]\left[\mathcal{M}_{S}\right]^{1 / 2}\right\}_{j j},
$$

which is, for eigenmode $j$, an equivalent diagonal damping rate due to the presence of the fuzzy substructures, i.e. the structural complexity.

(2)- There is an excellent agreement in terms of modulus and phase between the results given by the fuzzy structures theory (smoothed solid line) and the results of the numerical simulation of the fuzzy structure (irregular solid line). 


\section{CONCLUSION}

We have presented an explicit model of the generalized damping matrix due to structural complexity for the low frequency range. This model is deduced from the fuzzy structures theory previously developped by the author for the medium frequency range and can easily be implemented in the finite element codes. The model proposed yields a full generalized damping matrix whose its entries are explicitly known as a function of the geometry of the fuzzy substructures boundaries, the trace of the eigenmodes on these boundaries and the mean parameters of the fuzzy substructures. For applications these mean parameters are generally known except the mean coefficients of participating fuzzy mass which are dimensionless parameters. Therefore, these parameters must be updated and we has shown that this updating can be obtained solving a constrained optimization problem which is an usual quadratic programming problem. Finally, we have presented an example which contributes to the validation of the theory proposed and which cleary shows the physical role plays by the structural complexity in the vibration damping of the master structure.

\section{REFERENCES}

1 C. Soize, "Theoretical study of the probabilistic fuzzy", Technical Report ONERA 45-3454RY053R (in french), ONERA, Chatillon, France (1985).

2 C. Soize, "Probabilistic structural modeling in linear dynamic analysis of complex mechanical systems. I - Theoretical elements", La Recherche Aérospatiale (English edition) 5, 23-48 (1986).

3 F. Chabas and C. Soize, "Numerical study of a fuzzy probabilistic constitutive law", Technical Report ONERA 46-3454RY053R (in french), ONERA, Chatillon, France (1985).

4 F. Chabas, A. Desanti and C. Soize, "Probabilistic structural modeling in linear dynamic analysis of complex mechanical systems. II - Numerical analysis and applications", La Recherche Aérospatiale (English edition) 5, 49-67 (1986).

5 F. Chabas and C. Soize, "Modeling mechanical subsystems by boundary impedance in the finite element method", La Recherche Aérospatiale (English edition) 5, 59-75 (1987).

6 C. Soize, "Medium frequency linear vibrations of anisotropic elastic structures", La Recherche Aérospatiale (English edition) 5, 65-87 (1982).

7 C. Soize, P.M. Hutin, A. Desanti, J.M. David and F. Chabas, "Linear dynamic analysis of mechanical systems in the medium frequency range", Computers and Structures 23(5), 605-637 (1986).

8 F. Chabas and C. Soize, "Hydroelasticity of slender bodies in an unbounded fluid in the medium frequency range", La Recherche Aérospatiale (English edition) 4, 39-51 (1986).

9 C. Soize, J.M. David and A. Desanti, "Dynamic and acoustic response of coupled structure - dense fluid axisymmetric systems excited by a random wall pressure field", La Recherche Aérospatiale (English edition) 5, 1-14 (1989).

10 C. Soize, "Numerical methods in elastoacoustic in the non-modal domain", Proceedings of the international symposium on " Prediction of the noise emitted by vibrating structures", CETIM-Senlis, France, March26-28 1991, Revue française de mécanique, Special issued number 1991, 412-437 (1991).

11 C. Soize, A. Desanti and J.M. David, "Numerical methods in elastoacoustic for low and medium frequency ranges", La Recherche Aérospatiale (English edition) 5, (1992).

12 F. Chabas, A. Desanti and C. Soize, "Acoustic scattering from submerged elastic structures", Technical Report ONERA 59-3454RY067R (in french), ONERA, Chatillon, France (1987). 
D. Felix and A. Desanti, "Acoustic scattering from an submerged elastic structure. Comparison between numerical model and experiments", Technical Report ONERA 80-3454RY175R (in french), ONERA, Chatillon, France (1988).

14 R. Vasudevan, "Solution of acoustic problems using time integration and transform techniques", David Taylor Research Center, DTRC Report SAD-91/20e-1941, (1991).

R. Vasudevan and Y.N.Liu, "Application of time integration and transform techniques to scattering problems", NCA-12/AMD-128, Structural Acoustics, ASME, 35-40 (1991).

V.W. Sparrow, "Soize's theory of structural fuzzy : an examination of fundamental assumptions", Paper 2SA3, 121st meeting of ASA, Baltimore, MD (1991).

17 D.A. Russell and V.W. Sparrow, "Acoustic scattering from a fluid loaded plate with an attached structural fuzzy", Paper 7S11, 123rd meeting of ASA, Salt Lake City, Utah (1992).

18 A.D. Pierce, V.W. Sparrow and D.A. Russell, "Fundamental structural-acoustic idealizations for structures with fuzzy internals", ASME Winter Annual Meeting, New Orleans, Louisiana, Nov. 28 - Dec. 3 (1993).

19 A.D. Pierce, "Mass per unit natural frequency as a descriptor of internal fuzzy structure", $J$. Acoust. Soc. Am. 95(5), Pt. 2, 2845-2845 (1994).

20 M. Strasberg and D. Feit, "Vibration damping of large structures induced by attached small substructures each with multiple degrees of freedom", J. Acoust. Soc. Am. 95(5), Pt. 2, 2846-2846 (1994).

21 D.A. Russell, V.W. Sparrow and C. Soize, "A mathematical formulation for modeling the type I fuzzy parametres for a continuous line fuzzy attachment", J. Acoust. Soc. Am. 95(5), Pt. 2, 2846-2846 (1994).

J.J. McCoy, "The theory of fuzzy structures - A statistical continuum mechanics interpretation" J. Acoust. Soc. Am. 95(5), Pt. 2, 2846-2846 (1994).

23 J.L. Rochat and V.W. Sparrow, "The effects of fuzzy attachments on compressional and shear waves in a plate", J. Acoust. Soc. Am. 95(5), Pt. 2, 2847-2847 (1994).

24 I. Dyer, "Scattering from internally complex finite shell models", J. Acoust. Soc. Am. 95(5), Pt. 2, 2867-2867 (1994).

25 C. Soize, "A model and numerical method in the medium frequency range for vibroacoustic predictions using the theory of structural fuzzy", J. Acoust. Soc. Am. 94(2), Pt. 1, 849-865 (1993).

26 F. Chabas and C. Soize, "Etude et construction d'une loi de comportement du flou structural avec mémoire spatiale", Technical Report ONERA 64-3454RY160R, ONERA, Chatillon, France (1987).

27 C. Soize, "Modeling and numerical method in the medium frequency range for vibroacoustic predictions using the theory of structural fuzzy", J. Acoust. Soc. Am. 92(4), Pt. 2, 2365-2365 (1992).

28 D.M. Himmelblan, Applied Nonlinear Programming, McGraw-Hill, New York (1972). 


\section{LEGENDS ACCOMPANYING EACH FIGURE}

FIG. 1. Geometry of the master structure with its fuzzy substructure.

FIG. 2. Function $\mathcal{J}(\xi, \tau, \alpha)$ (eqn. (14)) versus $\tau$ for $\xi: 0.0001$ (thin line), 0.001 (thick line), 0.01 ( thin brokenline), 0.1 (thick brokenline), and for $\alpha=1$ fig. 2. (a) and $\alpha=0.1$ fig. 2 . (b).

FIG. 3. Explicative diagram yielding the attachment location and the eigenfrequency of each oscillator for a homogeneous fuzzy substructure simulated with simple linear oscillators. Each circle represents a linear oscillator whose its eigenfrequecy and its attachment location are uniformly distributed in each small rectangle.

FIG. 4. Geometry of the master structure (simply supported beam having 20 meters length) with two fuzzy substructures in zones defined by $4 \leq x_{1} \leq 8$ and $12 \leq x_{1} \leq 18$. Excitation in bending mode in $\left(x_{1}, x_{3}\right)$ plane is done by a unit point force applied at $x_{1}=9$.

FIG. 5. Eigenfrequency and spatial distribution of the linear oscillators for fuzzy substructures $\Gamma_{1}$ and $\Gamma_{2}$.

FIG. 6. Functions $\omega \mapsto h_{3}^{1}(\omega)$ and $\omega \mapsto h_{3}^{2}(\omega)$ defined by eqn. (13).

FIG. 7. Modulus (a), real part (b) and imaginary part (c) of the transversal accceleration of the beam at $x_{1}=9$ for, the beam with no oscillator (dashed line), the beam with the oscillators (irregular solid line) and the beam with oscillators modelized with the fuzzy structures theory (smoothed solid line).

FIG. 8. Modulus (a), real part (b) and imaginary part (c) of the transversal accceleration of the beam at $x_{1}=14$ for, the beam with no oscillator (dashed line), the beam with the oscillators (irregular solid line) and the beam with oscillators modelized with the fuzzy structures theory (smoothed solid line).

FIG. 9. Equivalent diagonal damping rate due to the fuzzy substructures and defined by eqn. (54). 